\title{
Effects of high-intensity focused ultrasound treatment on peripancreatic arterial and venous blood vessels in pancreatic cancer
}

\author{
XIAOYIN GUO ${ }^{1}$, HUI ZHU ${ }^{2}$, KUN ZHOU $^{1,2}$, CHENGBING JIN $^{2}$, YANG YANG $^{3}$, JUN ZHANG $^{2}$, \\ WEI YANG ${ }^{2}$, LIFENG RAN ${ }^{2}$ and DOBROMIR DIMITROV DIMITROV ${ }^{4}$
}

\begin{abstract}
${ }^{1}$ State Key Laboratory of Ultrasound Engineering in Medicine Co-Founded by Chongqing and the Ministry of Science and Technology, Chongqing Key Laboratory of Ultrasound in Medicine and Engineering, College of Biomedical Engineering, Chongqing Medical University, Chongqing 400016; ${ }^{2}$ Clinical Center for Tumor Therapy; ${ }^{3}$ Department of Ultrasound, The Second Affiliated Hospital of Chongqing Medical University, Chongqing 400010, P.R. China;

${ }^{4}$ Department of Surgery, Medical University Pleven, Pleven 5800, Bulgaria
\end{abstract}

Received September 11, 2019; Accepted February 11, 2020

DOI: $10.3892 / 01.2020 .11511$

\begin{abstract}
The present study aimed to evaluate the safety of high-intensity focused ultrasound (HIFU) treatment on peripancreatic arterial and venous blood vessels in patients with pancreatic cancer. This trial included 15 patients with pancreatic cancer ( 9 females and 6 males; age, 39-81 years; median age, 62 years). All patients underwent preoperative computed tomography (CT) or magnetic resonance imaging (MRI) and color Doppler flow imaging (CDFI) to assess the vascular hemodynamics of peripancreatic arterial and venous blood vessels pre-treatment. These patients were re-examined within 1 week post-HIFU treatment. Then, vascular adverse events were observed and followed up clinically. Prior to HIFU treatment, vessel involvement was recorded in 13 patients, including tumor lesions invading 19 veins and 14 arteries, which refers to the growth of pancreatic tumor lesions surrounding blood vessels, or tumor growth into blood vessels. In addition, 9 veins and 13 arteries were $<1 \mathrm{~cm}$ from the lesions. The hemodynamic parameters of peripancreatic vessels were measured using CDFI, including mean blood flow velocity, peak systolic blood flow velocity, vascular resistance index, vascular pulsatility index, vascular diameter, vascular blood flow and other indicators, to assess vascular perfusion in CT/MRI. There were no significant differences in preoperative and postoperative hemodynamic data $(\mathrm{P}>0.05)$. Overall, HIFU demonstrated no negative effects on peripancreatic arterial and venous blood vessels
\end{abstract}

Correspondence to: Professor Kun Zhou,Clinical Center for Tumor Therapy, The Second Affiliated Hospital of Chongqing Medical University, 74 Linjiang Road, Chongqing 400010, P.R. China E-mail: zhoukun@hospital.cqmu.edu.cn

Key words: pancreatic cancer, high-intensity focused ultrasound, vascular adverse events in patients with pancreatic cancer, even with tumor lesions wrapped in blood vessels. In addition, no complications of vascular stenosis and vascular adverse events were observed in the present study.

\section{Introduction}

Pancreatic carcinoma is a highly malignant gastrointestinal cancer with a poor prognosis and a low 5-year survival rate (1). Recently, the incidence and mortality rates of pancreatic cancer have steadily increased in the United States, within Europe and in China (1-4). According to the American Cancer Society, there were $\sim 55,440$ novel pancreatic cancer diagnoses and $\sim 4,330$ pancreatic cancer-associated mortalities in 2018 in the USA $(4,5)$. By 2030, pancreatic cancer is expected to become the second leading cause of cancer-associated mortality in the USA (1). Meanwhile, in 2015, the China Cancer Center demonstrated that pancreatic cancer ranks 8th in terms of incidence among male patients with a malignancy (2). The mortality rate of all types of cancer in Beijing and Shanghai ranks fifth (2,3). Pancreatic cancer often presents with lesions that invade adjacent blood vessels, such as mesenteric and splenic vessels, with the patient losing the opportunity of surgical resection (6). Therefore, patients with pancreatic cancer have limited treatment options and a poor quality of life. To improve the quality of life and prolong survival in such patients, local ablative procedures are used to treat pancreatic carcinoma (7). These treatments include irreversible electroporation (IRE) (8), radiofrequency ablation (RFA) (9) and high-intensity focused ultrasound (HIFU) (10), which have been widely used in the past few years, achieving good therapeutic effects in pancreatic carcinoma.

HIFU is a non-invasive procedure for the ablative treatment of localized tumors. The basic principle is that ultrasound is focused at the focal region and produces biological effects, including thermal, cavitation and mechanical effects, to achieve thermal ablation of the target tissue, with pathological changes, including coagulative necrosis (11). HIFU has been 
widely used in the treatment of uterine fibroids and hepatocellular carcinoma, with good therapeutic results (12). Previous studies have confirmed that HIFU therapy effectively alleviates cancer-associated abdominal pain, reduces tumor volume and may confer an additional survival benefit (11,13-23). However, vascular complications caused by this treatment have been reported, including secondary occlusion of the superior mesenteric artery (24) and portal vein thrombosis (25). The present study aimed to evaluate the safety of HIFU therapy by assessing blood vessel events in patients with pancreatic cancer.

\section{Materials and methods}

Patients and lesions. The present observational single-center study was approved by The Ethics Committee of the Second Affiliated Hospital of Chongqing Medical University (Chongqing, China; approval no. 12/2018). Every patient provided written informed consent before treatment initiation. Between April 2018 and April 2019, 15 patients with pancreatic carcinoma (Union for International Cancer Control (UICC) stage II-IV) (6) were enrolled, including 6 males and 9 females (mean age, 39-81 years; median age, $65 \pm 11$ years). According to the TNM staging system (6), a total of two patients were diagnosed as stage II, six patients as stage III and seven patients were diagnosed as stage IV. Of the 15 patients, 7 had tumors of the pancreatic head and 8 had tumors in the body and/or tail of the pancreas. The inclusion criteria were: i) Confirmed diagnosis of pancreatic carcinoma (6); ii) tumor invading a blood vessel; and iii) distance between the pancreatic tumor and peripancreatic blood vessel $<1 \mathrm{~cm}$. The exclusion criteria were: i) Non-eligibility for general anesthesia; ii) calcification of blood vessels invaded by tumor lesions; iii) Child-Pugh class C (26); iv) hemorrhage and other severe diseases, such as severe heart failure, cerebrovascular disease and renal insufficiency and v) poorly managed diabetes.

All patients underwent $\mathrm{CT}$ or MRI and were assessed using color Doppler flow imaging (CDFI) to evaluate the vascular hemodynamic changes of peripancreatic arterial and venous vessels pre-treatment and at 1 week and 1-month post-HIFU treatment. The primary blood vessels around the pancreas included the splenic artery, splenic vein, superior mesenteric artery, superior mesenteric vein (SMV), hepatic artery, celiac artery and portal vein (PV). The hemodynamic parameters of each blood vessel were measured using CDFI, including mean blood flow velocity, peak systolic blood flow velocity (PSV), vascular resistance index (RI), vascular pulsatility index (PI), vascular diameter and vascular blood flow. Combined with pre- and post-HIFU treatment imaging modalities, such as MRI and CT, changes in blood vessel shape and inner diameter were assessed and vessel occlusion, thrombosis and hemorrhage were recorded.

Color Doppler ultrasound examination. An APLI0 500TUS-A500 color Doppler ultrasound system (TOSHIBA) was used with a wide-band convex array probe with a center frequency of $3.5 \mathrm{MHz}$ and a mechanical index of 0.06-0.15.

Ultrasound examination was performed by an experienced sonographer. All patients with pancreatic cancer underwent ultrasound examination in the fasting state, using a 3.0-5.0 MH variable convex transducer. The operating steps were similar to the routine abdominal scan method (27), followed by abdominal parenchymal organs, the biliary system and retroperitoneal large blood vessels and their primary branches. There was final focus on the location, size, echo, boundary and blood flow of the lesion. Image quality was improved by excluded artifact interference, adopting an appropriate graded compression scan and focusing on observation and recording the relationship between pancreatic tumor lesions and blood vessels around the pancreas. The clearest slice was selected and the probe was fixed. The mean blood flow rate, systolic blood flow velocity peak, vascular resistance index, vascular pulsation index and vessel diameter at the closest point from the deep surface of the tumor lesion were measured.

HIFU therapy. HIFU was performed using a Model-JC Focused Ultrasound Tumor Therapeutic system (Chongqing Haifu Medical Technology Co., Ltd.) equipped with a diagnostic ultrasound probe $(3.50-5.00 \mathrm{MHz})$ for real-time guidance and a therapeutic transducer (focal length, 10-25; diameter, $10-30 \mathrm{~cm}$ ) operating at 0.5-2 MHz. The focal region was an ellipsoid with short and long axes of 3 and $8 \mathrm{~mm}$, respectively.

Prior to HIFU treatment, all patients underwent colonic lavage with liquid food, laxatives and cleansing enema to protect the gastrointestinal tract in front of the target area. The gastric tube was then placed and gastric juice changes were observed with a vacuum suction device to prevent gastrointestinal damage and reduce the occurrence of postoperative pancreatitis, until 1-2 days after surgery. Skin preparation in the treated area was performed by degreasing with $75 \%$ ethanol and degassing with a vacuum aspirator to avoid skin burns. During HIFU treatment, the patient was placed in the prone position after general anesthesia. According to the proposed HIFU treatment plan, real-time ultrasound monitoring was used to determine tumor location and size, carefully identifying lesions invading blood vessels, as shown in Fig. 1.

The lesions were divided into slices 5-mm-thick and focus was placed on the deepest layer of a slice containing the maximum tumor area. A safety gap of $\sim 15 \mathrm{~mm}$ was maintained in case the target area was close to important organs, including large blood vessels or the gastrointestinal tract and near the tumor edge. The target area of the tumor lesion was locally ablated from the deepest region to the surface and the treatment was repeated for each slice until the tumor was completely ablated. In the course of treatment, point scan was the main method at a power of 100-400 W. Notable signs of effective HIFU sonication included the presence of massive gray-scale changes (MGSCs) or an increase in gray-scale throughout the target area. Treatment was complete when the tumor volume was fully covered by MGSCs. The treatment parameters and patient characteristics are shown in Table I.

Data collection. A total of 15 patients with pancreatic cancer were treated with HIFU. According to the imaging manifestations of pancreatic cancer invading peripancreatic blood vessels, the relationship between blood vessels and the tumor were divided into two groups: i) Normal pancreatic tissue between the tumor and blood vessels, with the tumor $<1 \mathrm{~cm}$ from major blood vessels; and ii) tumor adjacent to or 
Table I. Baseline characteristics of patients with pancreatic cancer and who underwent high-intensity focused ultrasound treatment.

\begin{tabular}{lc}
\hline Characteristic & $\mathrm{n}$ \\
\hline Total number of patients & 15 \\
Sex & \\
Male & 6 \\
Female & 9 \\
Age, years (median \pm SD) & $65 \pm 11$ \\
Site of pancreatic disease & \\
Head & 7 \\
Body or tail & 8 \\
CA19-9 & \\
+ & 15 \\
- & 0 \\
TNM stage & 2 \\
II & 6 \\
III & 7 \\
IV & $81 \pm 37$ \\
Intervention duration, min (mean \pm SD) & $725 \pm 370$ \\
Therapeutic sonication duration, & \\
sec (mean \pm SD) duration (seconds) & $295 \pm 54$ \\
Total energy, J (mean \pm SD) & $68.4 \%$ \\
Average power, W (mean \pm SD) & \\
Lesion ablation rate, $\%$ & \\
\hline
\end{tabular}

CA19-9, carbohydrate antigen 19-9; SD, standard deviation; TNM, Tumor-Node-Metastasis.

surrounding the blood vessel. The association between tumor and blood vessel was recorded. All patients underwent CT or MRI and underwent abdominal blood vessel CDFI to analyze hemodynamic parameters. The follow-up period of the present study was between June 2018 and September 2019. Follow-up examinations included abdominal blood vessel CDFI, and CT or MRI every 1 or 2 months.

Evaluation of therapeutic efficacy and pain relief. The treatment efficacy was evaluated using contrast enhanced CT or MRI 1 week post-HIFU treatment and pain relief pre-treatment and at 1 week after treatment was assessed using the NRS pain score table (numerical rating scale of $0-10$, with 0 indicating 'no pain' and 10 reflecting 'maximum imaginable pain') (7). Based on images obtained using contrast enhanced CT or MRI, no enhancement area was observed in the tumor lesion, which was considered to be completely ablated and necrotic. Blood vessel adverse events were defined as non-perfusion or partial perfusion. The rate of lesion ablation was calculated based on preoperative and postoperative contrast enhanced CT or MRI. 3D Image Processing software (version 1.0; Chongqing Haifu Medical Technology Co., Ltd.) was used to delineate the tumor and determine the tumor volume, non-perfused volume (NPV) and lesion ablation rate (\%) as NPV/tumor volume $\mathrm{x} 100$.

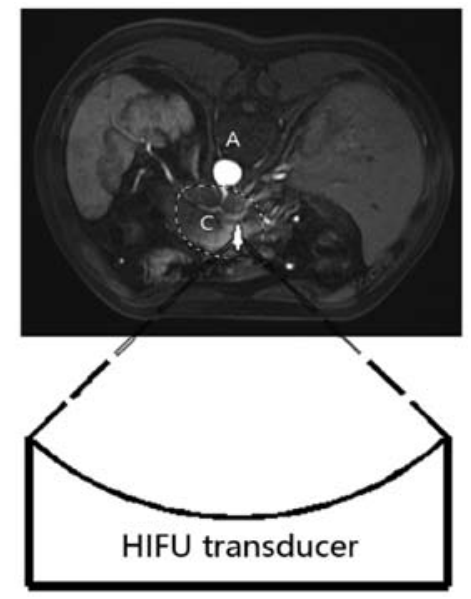

Figure 1. Schematic representation of patient positioning for high-intensity focused ultrasound ablation of pancreatic neoplasms. C, targeted pancreatic cancer; A, abdominal aorta; HIFU, high-intensity focused ultrasound.

Statistical analysis. Data were analyzed using SPSS version 22.0 software (IBM Corp.). Data are presented as the mean \pm standard deviation. Preoperative and postoperative samples were compared using a paired t-test. $\mathrm{P}<0.05$ was considered to indicate a statistically significant difference.

\section{Results}

Clinical characteristics. All patients successfully completed the HIFU treatment procedure for pancreatic cancer and no clinical complications, such as persistent severe abdominal pain, gastrointestinal bleeding, obstructive jaundice and peritoneal irritation, were observed. The specific invaded and adjacent blood vessels are presented in Table II. A total of 33 blood vessels were invaded by tumor lesion and 22 were within $1 \mathrm{~cm}$ of the lesion, which were well identified using imaging. Compared with preoperative NRS pain score $(4.0 \pm 2.0)$, postoperative NRS pain score (1.6 \pm 1.3$)$ was significantly reduced $(\mathrm{P}<0.01)$, indicating pain relief after the operation. The lesion ablation rate was $68.4 \%$, which was calculated using 3D image processing software, and the tumor marker CA19-9 was positive in all patients (Table I), which is useful for the diagnosis and treatment of the disease (6).

Hemodynamic data analysis. Specific arterial and venous hemodynamic parameters (PSV, MV, VF, PI, RI and VD) are presented in Tables III and IV. As presented in Table III, comparing the PI and RI of arterial vessels before and after HIFU treatment, there were no significant differences (P>0.05). Furthermore, there was no significant change in the PSV, MV, VF and VD of arterial blood vessels after HIFU compared with pre-treatment values $(\mathrm{P}>0.05)$. There was no significant change in the PSV, MV, VF and VD of venous blood vessels after HIFU compared with pre-treatment values (Table IV; P>0.05). These data indicated that the functions of these vessels had no obvious changes after HIFU treatment.

Adverse effects of HIFU treatment. The postoperative complications in patients included fever $(n=1)$ and skin numbness in the treatment area $(n=1)$. Both patients improved following 
Table II. Information on the association between tumor lesions and blood vessels in patients with pancreatic cancer, treated with high-intensity focused ultrasound $(n=15)$.

\begin{tabular}{lcc}
\hline Blood vessel & $\begin{array}{c}\text { Number } \\
\text { of vessels } \\
\text { invaded }\end{array}$ & $\begin{array}{c}\text { Number } \\
\text { of vessels } \\
\text { within } 1 \mathrm{~cm}\end{array}$ \\
\hline Splenic vein & 9 & 1 \\
Splenic artery & 7 & 3 \\
Superior mesenteric vein & 6 & 4 \\
Superior mesenteric artery & 3 & 6 \\
Portal vein & 4 & 4 \\
Celiac artery & 2 & 2 \\
Hepatic artery & 2 & 2 \\
Total & 33 & 22 \\
\hline
\end{tabular}

symptomatic treatment. No imaging changes of adjacent vessels, such as stenosis and occlusion, were observed in imaging data. No blood vessel adverse events were observed within 1 week after HIFU treatment and during follow-up. The results demonstrated that splenic vessels were the most frequently invaded blood vessels in pancreatic tumors (Table II). Following comparison of data from all patients, a 63-year-old female patient with pancreatic cancer, whose MRI showed that the tumor invaded the splenic vein and was adjacent to the splenic artery in the tail of the pancreas (Fig. 2Aa), which was similar to most pancreatic cancer patients in our study and had more representative data and had certain research value. Finally, the incidences of avascular adverse events and associated complications in pancreatic cancer treated with HIFU were lower compared with other local ablation methods, such as RFA and IRE (Table V).

\section{Discussion}

Pancreatic cancer is highly malignant and lacks a typical set of early stage symptoms. Most patients are diagnosed with advanced disease and are usually not eligible for surgical treatment due to tumor invasion of mesenteric roots and arterial vessels, or because of liver and peritoneal metastasis. As a result, the 5-year survival rate is very low and decreases year by year (6). As a non-invasive treatment modality, HIFU has achieved good therapeutic results in the treatment of various benign and malignant tumors. Asian and European patients benefit from survival and pancreatic cancer related pain relief after HIFU treatment (28). It was first reported in 2000 that HIFU successfully ablates and treats pancreatic cancer (29). Several studies have confirmed the safety and efficacy of HIFU in the treatment of pancreatic cancer (10,14-16,19-25,29-34). The most common complications observed following HIFU treatment of pancreatic cancer include skin burns $(10,15,17,21-23,30-32)$, pancreatitis $(10,18,23,31,32)$, duodenal fistula $(23,31)$ and obstructive jaundice $(19,34)$. Vascular adverse events occur infrequently, including vascular complications of secondary occlusion of superior mesenteric artery (24) and PV thromboses (25). To the best of our knowledge, no cases of vessel rupture and bleeding have been described. Previous studies have shown that adjacent blood vessels are safe from HIFU ablation of the tumors near large hepatic and PVs in the liver $(35,36)$. Zhang et al $(35)$ reported that HIFU could safely and effectively ablate lesions close to large blood vessels without damage to such vessels, with no blood vessel adverse events observed. In addition, HIFU ablation of pancreatic cancer is safe for peripancreatic blood vessels. Strunk et al (16) reported that $94 \%$ of patients showed no patency change in associated vessels after HIFU treatment of locally pancreatic cancer with tumor invasion and encasement of blood vessels. Meanwhile, no vascular adverse events were recorded.

The aforementioned studies focused on the imaging changes of blood vessels assessed using CT/MRI and did not assess vascular function using hemodynamics analysis using CDFI. The purpose of the present study was to evaluate the effect of HIFU treatment on vascular function by measuring preoperative and postoperative hemodynamic parameters of peripheral blood vessels in pancreatic cancer cases using CDFI, observing the shape changes of blood vessels using imaging, to determine potential adverse events of adjacent blood vessels after HIFU treatment of pancreatic cancer.

In the present study, vascular shape assessment in all patients revealed that splenic vessels, superior mesenteric vessels and PVs were mainly involved. Based on images assessing potential filling defects in the analyzed blood vessels, occlusion or thrombosis was ruled out. The blood vessels associated with the tumors were observed and their inner diameters were measured. Finally, the imaging data obtained before and after HIFU treatment were compared. There were no shape changes of blood vessels, as well as no vascular adverse events, such as vascular occlusion, thrombosis and rupture of blood vessels. These results corroborated previous studies $(16,35)$.

Based on the data obtained in the present study, hemodynamic parameters reflected the functions of peripancreatic blood vessels. Among hemodynamic indexes, PI and RI reflect the resistance of arterial vessels. Specifically, PI denotes blood vessel wall elasticity, while RI directly reflects resistance to blood flow (37). The elasticity of the associated vessels did not change significantly following HIFU treatment. PSV reflects the degree of vascular filling, indicating whether there is a change in blood supply to distal tissues and organs (38). In the present study, all patients received preoperative and postoperative CDFI examinations and the hemodynamic parameters of pancreatic tumor lesions and adjacent blood vessels were analyzed. In addition, a comparative analysis of venous and arterial blood vessels was performed. These data also indicated that HIFU treatment had no significant influence on the function of peripancreatic blood vessels and did not affect peripancreatic tissues or organs.

There were fewer complications and side effects after tumor lesion ablation in the present study compared with previous reports $(14,28,33,34,39,40)$. Meanwhile, no notable clinical symptoms and manifestations of mesenteric vascular occlusion, such as persistent abdominal pain and peritoneal irritation, were observed. Pain was significantly reduced in most patients after HIFU treatment, but acute pain occurred immediately post-HIFU in one patient. It was observed that the transient stimulation reaction of HIFU ablation to the 

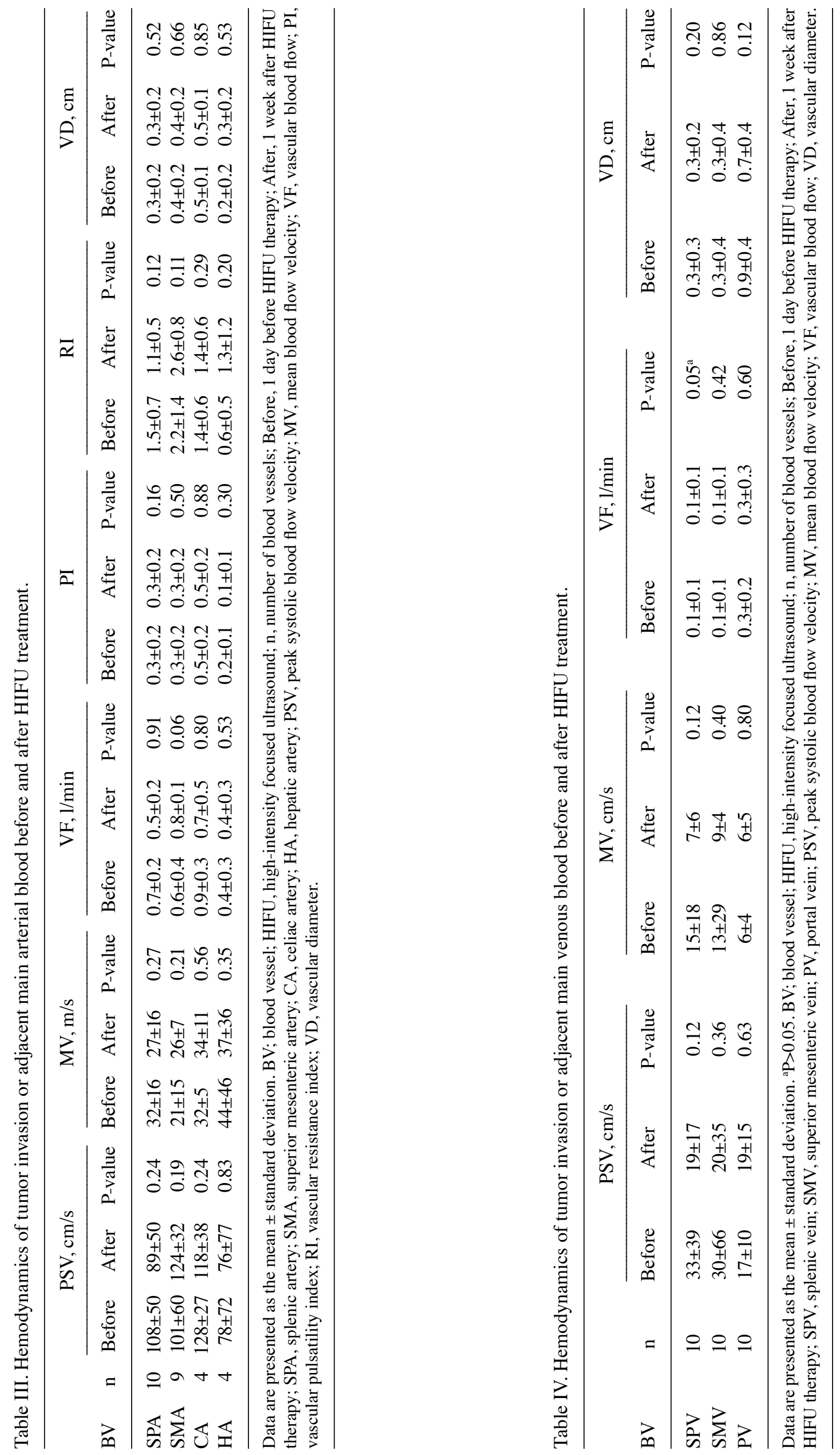
A a

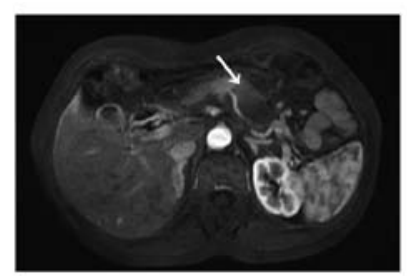

B a

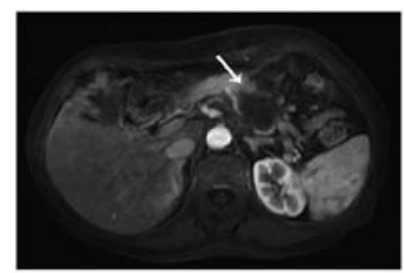

C a

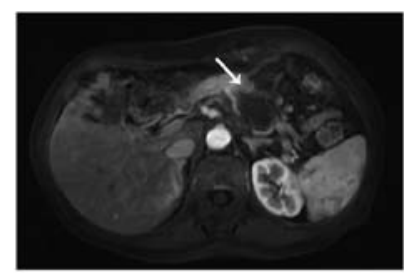

D a

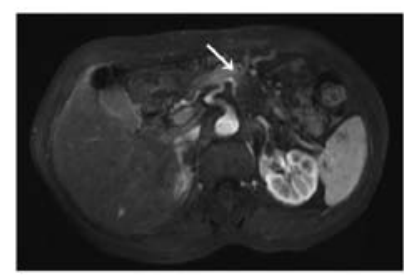

E

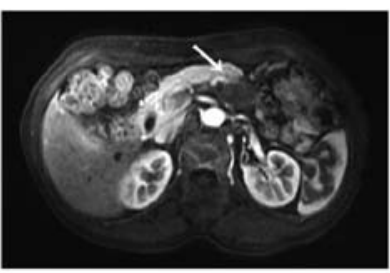

b

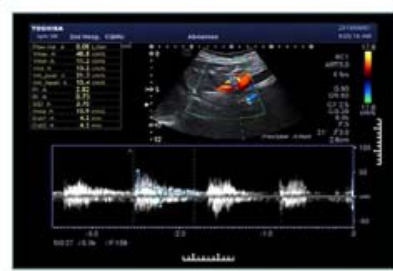

b

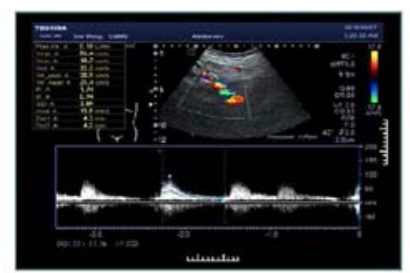

b

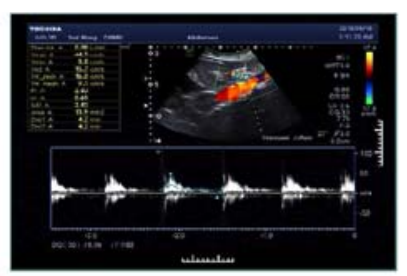

b

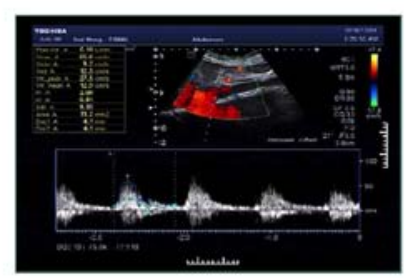

C

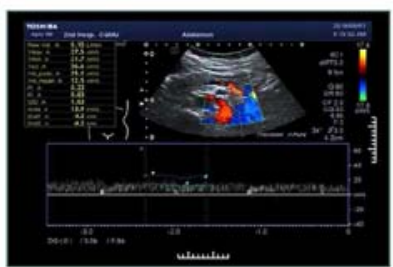

C

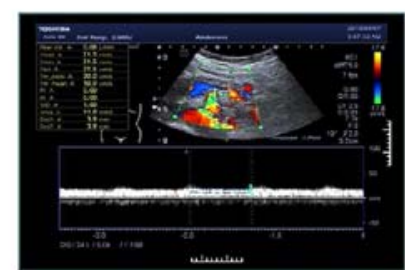

C

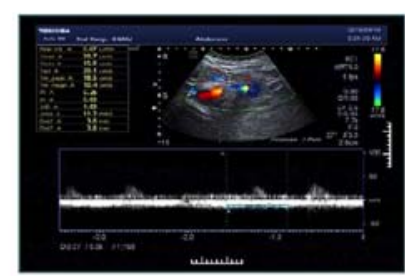

C

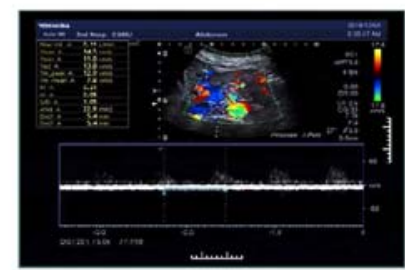

Figure 2. A 63-year-old female patient with pancreatic cancer in the tail of pancreas was evaluated radiologically (A) before HIFU, (B) at 1 week, (C) at 1 month, (D) at 4 months and (E) 10 months after HIFU, respectively. Arrows indicate a tumor lesion in the tail of the pancreas. Before HIFU, (Aa) enhanced MRI showed that the tumor invaded the splenic vein and was adjacent to the splenic artery. CDFI images showed normal blood flow in (Ab) splenic artery and (Ac) splenic vein. At 1week after HIFU, (Ba) enhanced MRI showed no significant change in the morphology of splenic blood vessels, and no overt abnormal changes of hemodynamics of the (Bb) splenic artery and (Bc) vein in CDFI images. Follow-up 1 month after HIFU, (Ca) enhanced MRI showed that splenic vessel filling was normal in the enhancement phase, with no vascular stenosis or occlusion. There was no significant change in hemodynamics of the $(\mathrm{Cb})$ splenic artery and $(\mathrm{Cc})$ vein. At 4 months after HIFU, (Da) the vascular morphology of the splenic vein and the splenic artery were normal, and the hemodynamics of the $(\mathrm{Db})$ splenic artery and (Dc) vein vessels were similar to those of the previous follow-up. At 10 months after HIFU, (E) enhanced MRI showed smaller lesion volumes compared with preoperative values, with no vascular stenosis or occlusion. There were no CDFI images due to insufficient intestinal preparation and intestinal gas interference. MRI, magnetic resonance imaging; HIFU, high-intensity focused ultrasound; CDFI, color doppler flow imaging.

pancreas improves after symptomatic analgesia and significantly reduced pain on postoperative day 2 . The NRS pain score was significantly reduced compared with the preoperative results. The present study hypothesized that HIFU ablation of pancreatic tumor lesions could control tumor growth and reduce tumor compression to relieve pain, whereas HIFU thermal ablation has been reported to cause damage to peripheral nerves of the pancreas, thereby blocking pain nerve impulses $(22,25,28)$.

In previous studies, it was observed that HIFU ablation of malignant tumors has some effects on adjacent tissues. In a study of preoperative HIFU ablation for borderline resectable pancreatic cancer, the patient underwent surgical resection 1 week after HIFU treatment (41). Wang et al (41) observed faintly yellow burn marks on the vessel wall adjacent to the tumor lesion (the anterior-lateral part of the junction of the portal vein and the SMV) and normal vasoactive activity. These results are similar to those previous experiments reporting that HIFU effectively causes coagulative necrosis of the tissue near large blood vessels and that ablation at $0-5 \mathrm{~mm}$ close to the blood vessel may cause damage to the vessel wall, but such damage is reversible and could self-resolve within about 1 week (36). The present study separately evaluated the hemodynamic parameters of adjacent arterial and venous blood vessels, as well as vascular function. 


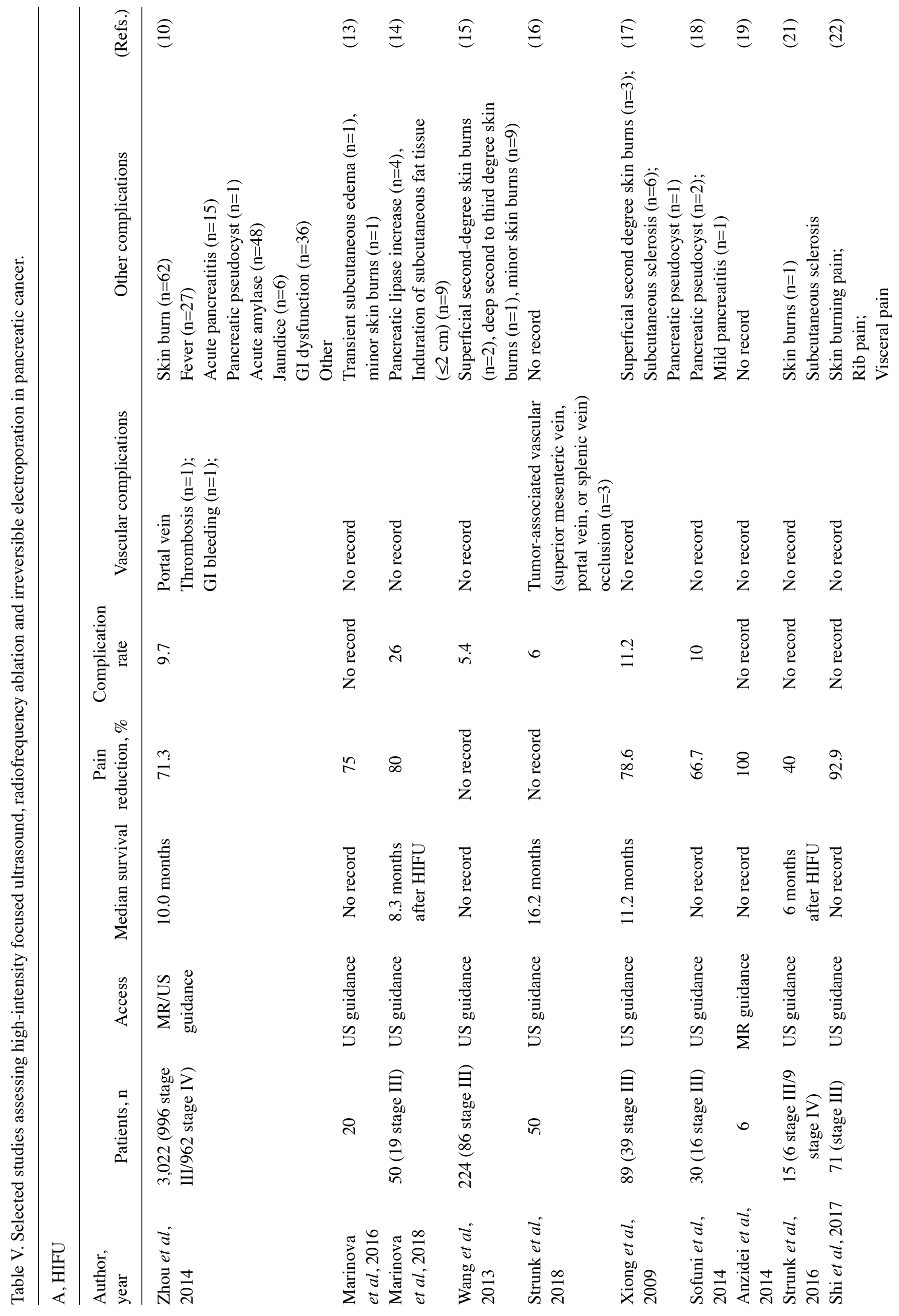




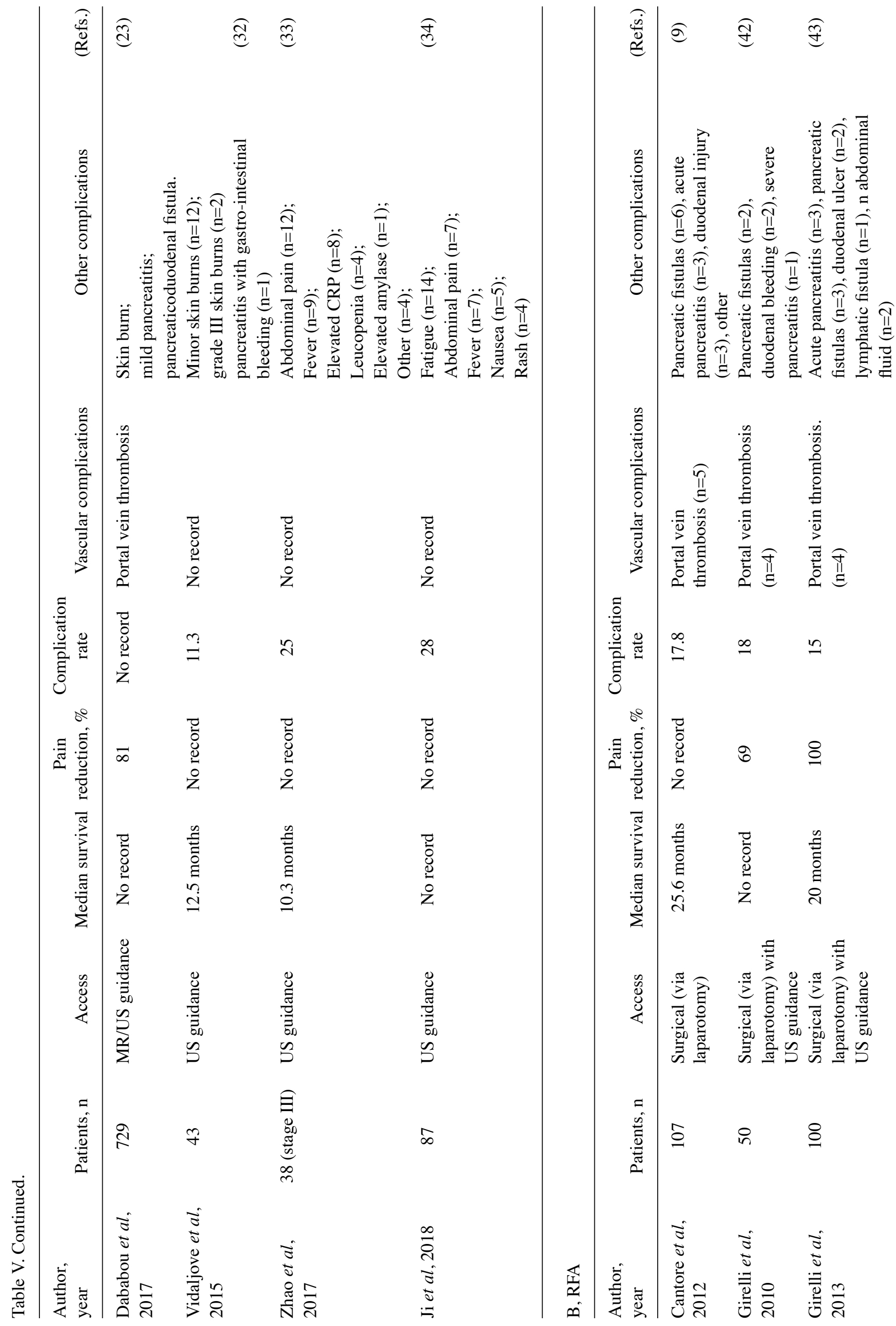




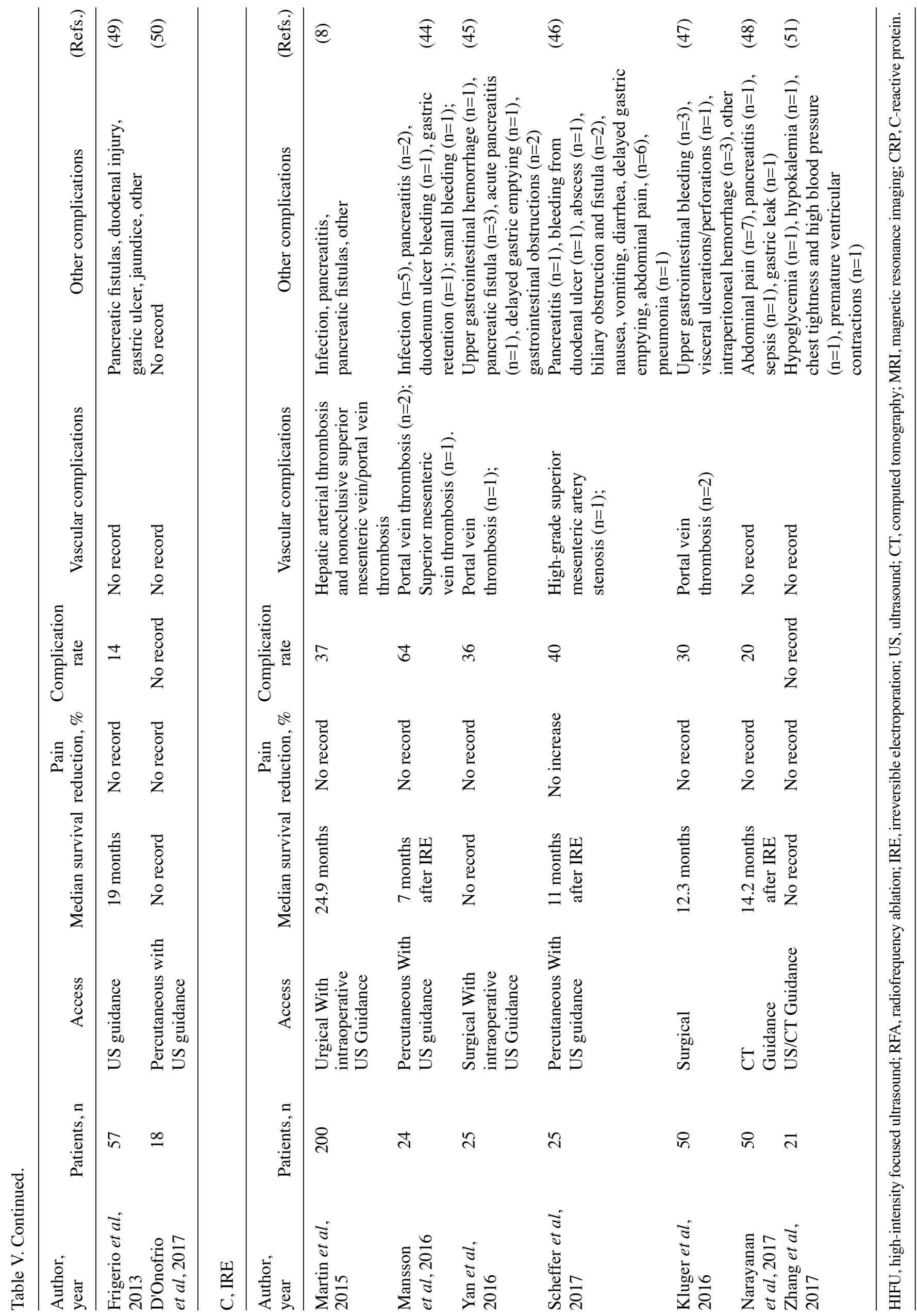


It was hypothesized that weak venous vessel wall and tumor compression or invasion of the vein slows the blood flow rate, increasing the likelihood of thrombosis (25). However, the blood flow rate of arterial vessels is high, combined with good vascular elasticity. Compared with venous vessels, arterial vessels have lower probability of thrombosis and partial narrowing of blood vessels is more likely to occur. This is consistent with findings from Strunk et al (16). In addition, physicians should be aware of the tumor squeezing blood vessels or tumor invasion of blood vessels during the HIFU therapy. It is understood that tumor compression or invasion of blood vessels reduces blood flow rate and favors coagulation (25). Meanwhile, the compression effect of contraction on blood vessels after tumor ablation, as well as the cavitation effect caused by ultrasound radiation, could damage the intima of blood vessels, inducing vasospasm and thrombosis and eventually causing partial occlusion of blood vessels (18).

Compared with other local ablation methods (RFA and IRE), HIFU has lower incidence rates of vascular adverse events and associated complications in pancreatic cancer. Portal vein thrombosis is the most common vascular-associated complication of pancreatic cancer following RFA and IRE treatment $(8,9,42-51)$. In related studies, patients with pancreatic cancer received RFA and IRE and the primary complications included acute pancreatitis $(8,9,42-46,48)$, abdominal pain $(46,48)$, pancreatic fistula $(8,9,42,43,45,49)$, duodenal ulcer or perforation $(9,42-44,49)$, gastrointestinal bleeding $(42,44-47)$ and biliary fistula (46). In HIFU, the main complications included acute pancreatitis, skin burn, abdominal pain $(22,33,34)$ and elevated amylase expression levels $(14,21,33,34)$, whereas vascular adverse events are rarely recorded and patients can recover and be discharged within a short time $(24,25)$. In addition, HIFU treatment utilizes ultrasound without involving the use of needles, electrodes, probes or similar items, therefore HIFU is safer and less invasive compared with other local ablation methods and can be performed in patients with tumors near vessels, the intestine or the bile duct stent (7). In addition, HIFU treatment could avoid potential complications caused by puncture, especially bleeding and metastasis in the puncture channel (16). Therefore, HIFU ablation of pancreatic cancer treatment is beneficial.

The present study was limited by the small number of patients. The morbidity of pancreatic cancer is relatively lower compared with other gastrointestinal malignancies, therefore there were not large numbers of patients with pancreatic cancer suitable to be involved in the present single-center study. Future studies should include a larger number of selected patients. For example, tumor size and location were not considered as exclusion criteria and vascular hemodynamic data immediately after surgery were not available due to general anesthesia. Due to the short follow-up time of the present study, the long-term survival rate of patients was not assessed.

In conclusion, the present single-center study assessed the shape and hemodynamics of related vessels before and after HIFU treatment and no significant changes were found, with blood vessels maintaining their normal function. No adverse vascular events were associated with HIFU therapy in pancreatic cancer. Therefore, it was concluded that HIFU therapy for pancreatic cancer has no deleterious effects on peripancreatic arterial and venous blood vessels.

\section{Acknowledgments}

Not applicable.

\section{Funding}

This study was financially supported by the Yuzhong District Research Program of Basic Research and Frontier Technology of Chongqing (grant no. 20170412), the Chongqing Research Program of Special Program Social Insurance and People's Livelihood (grant no. cstc2017shmsA130027) and the Chongqing Research Program of Clinical Evaluation and Application Demonstration of Innovative Medical Devices (grant no. cstc2015jcsf10002-14).

\section{Availability of data and materials}

The datasets used and/or analyzed during the current study are available from the corresponding author on reasonable request.

\section{Authors' contributions}

$\mathrm{XG}, \mathrm{HZ}, \mathrm{KZ}$ and DDD designed the present study. XG, YY, JZ, WY and LR performed the literature review. XG and CJ analyzed the data. XG and $\mathrm{KZ}$ drafted the initial manuscript. All authors read and approved the final manuscript.

\section{Ethics approval and consent to participate}

The present study was approved by the Ethics Committee of The Second Affiliated Hospital of Chongqing Medical University (Chongqing, China) and performed in accordance with The Declaration of Helsinki. Written informed consent was provided by all patients prior to the study start (approval no. 12/2018).

\section{Patient consent for publication}

Not applicable.

\section{Competing interests}

The authors declare that they have no competing interests.

\section{References}

1. Kamisawa T, Wood LD, Itoi T and Takaori K: Pancreatic cancer. Lancet 388: 73-85, 2016.

2. Chen W, Zheng R, Baade PD, Zhang S, Zeng H, Bray F, Jemal A, Yu XQ and He J: Cancer statistics in China, 2015. CA Cancer J Clin 66: 115-132, 2016.

3. Chen W, Sun K, Zheng R, Zeng H, Zhang S, Xia C, Yang Z, Li H, Zou X and He J: Cancer incidence and mortality in China, 2014 Chin J Cancer Res 30: 1-12, 2018.

4. Ashktorab H, Kupfer SS, Brim H and Carethers JM: Racial disparity in gastrointestinal cancer risk. Gastroenterology 153: 910-923, 2017.

5. Siegel RL, Miller KD and Jemal A: Cancer statistics, 2018. CA Cancer J Clin 68: 7-30, 2018.

6. Chun YS, Pawlik TM and Vauthey JN: 8th Edition of the AJCC cancer staging manual: Pancreas and hepatobiliary cancers. Ann Surg Oncol 25: 845-847, 2017.

7. Rombouts SJ, Vogel JA, van Santvoort HC, van Lienden KP, van Hillegersberg R, Busch OR, Besselink MG and Molenaar IQ: Systematic review of innovative ablative therapies for the treatment of locally advanced pancreatic cancer. Br J Surg 102: 182-193, 2015. 
8. Martin RC II, Kwon D, Chalikonda S, Sellers M, Kotz E, Scoggins C, McMasters KM and Watkins K: Treatment of 200 locally advanced (stage III) pancreatic adenocarcinoma patients with irreversible electroporation: Safety and efficacy. Ann Surg 262: 486-494; discussion 492-494, 2015.

9. Cantore M, Girelli R, Mambrini A, Frigerio I, Boz G, Salvia R, Giardino A, Orlandi M, Auriemma A and Bassi C: Combined modality treatment for patients with locally advanced pancreatic adenocarcinoma. Br J Surg 99: 1083-1088, 2012.

10. Zhou Y: High-intensity focused ultrasound treatment for advanced pancreatic cancer. Gastroenterol Res Pract 2014: 1-11, 2014.

11. Wu F: High intensity focused ultrasound: A noninvasive therapy for locally advanced pancreatic cancer. World J Gastroenterol 20 : $16480-16488,2014$

12. Wu F, Wang ZB, Chen WZ, Wang W, Gui Y, Zhang M, Zheng G, Zhou Y, Xu G, Li M, et al: Extracorporeal high intensity focused ultrasound ablation in the treatment of 1,038 patients with solid carcinomas in China: An overview. Ultrason Sonochem 11: 149-154, 2004

13. Marinova M, Strunk HM, Rauch M, Henseler J, Clarens T, Brüx L, Dolscheid-Pommerich R, Conrad R, Cuhls H, Radbruch L, et al: High-intensity focused ultrasound (HIFU) for tumor pain relief in inoperable pancreatic cancer: Evaluation with the pain sensation scale (SES). Schmerz 31: 31-39, 2016 (In German).

14. Marinova M, Huxold HC, Henseler J, Mücke M, Conrad R, Rolke R, Ahmadzadehfar H, Rauch M, Fimmers R, Luechters G, et al: Clinical effectiveness and potential survival benefit of US-guided high-intensity focused ultrasound therapy in patients with advanced-stage pancreatic cancer. Ultraschall Med 40: 625-637, 2019.

15. Wang K, Zhu H, Meng Z, Chen Z, Lin J, Shen Y and Gao H: Safety evaluation of high-intensity focused ultrasound in patients with pancreatic cancer. Onkologie 36: 88-92, 2013.

16. Strunk H, Lützow, Henseler J, Mücke M, Rauch M, Marx C, Schild $\mathrm{HH}$ and Marinova M: Mesenteric vessel patency following HIFU therapy in patients with locally invasive pancreatic cancer. Ultraschall Med 39: 650-658, 2018.

17. Xiong LL, Hwang JH, Huang XB, Yao SS, He CJ, Ge XH, Ge HY and Wang XF: Early clinical experience using high intensity focused ultrasound for palliation of inoperable pancreatic cancer. JOP 10: 123-129, 2009.

18. Sofuni A, Moriyasu F, Sano T, Itokawa F, Tsuchiya T, Kurihara T, Ishii K, Tsuji S, Ikeuchi N, Tanaka R, et al: Safety trial of high-intensity focused ultrasound therapy for pancreatic cancer. World J Gastroenterol 20: 9570-9577, 2014.

19. Anzidei M, Marincola BC, Bezzi M, Brachetti G, Nudo F, Cortesi E, Berloco P, Catalano C and Napoli A: Magnetic resonance-guided high-intensity focused ultrasound treatment of locally advanced pancreatic adenocarcinoma: Preliminary experience for pain palliation and local tumor control. Invest Radiol 49: 759-65, 2014.

20. Shi Y, Ying X, Hu X, Zhao J, Fang X, Wu M, Chen TZ and Shen $\mathrm{H}$ : Influence of high intensity focused ultrasound (HIFU) treatment to the pancreatic function in pancreatic cancer patients. Pak J Pharm Sci 28 (Suppl 3): S1097-S1100, 2015.

21. Strunk HM, Henseler J, Rauch M, Mücke M, Kukuk G, Cuhls H, Radbruch L, Zhang L, Schild HH and Marinova M: Clinical use of high-intensity focused ultrasound (HIFU) for tumor and pain reduction in advanced pancreatic cancer. Rofo 188: 662-670, 2016.

22. Shi Y, Ying X, Hu X and Shen H: Pain management of pancreatic cancer patients with high-intensity focused ultrasound therapy. Pak J Pharm Sci 30 (Suppl 1): S303-S307, 2017.

23. Dababou S, Marrocchio C, Rosenberg J, Bitton R, Pauly KB Napoli A, Hwang JH and Ghanouni P: A meta-analysis of palliative treatment of pancreatic cancer with high intensity focused ultrasound. J Ther Ultrasound 5: 9, 2017.

24. Wei W, Jie T and Huiyi Y: Ablation effects of high-intensity focused ultrasound therapy on pancreatic cancer. Chin J Ultrasound Med, 76-79, 2007.

25. Orsi F, Zhang L, Arnone P, Orgera G, Bonomo G, Vigna PD, Monfardini L, Zhou K, Chen W, Wang Z and Veronesi U: High-intensity focused ultrasound ablation: Effective and safe therapy for solid tumors in difficult locations. AJR Am J Roentgenol 195: W245-W252, 2010.

26. Christensen E, Schlichting P, Fauerholdt L, Gluud C, Andersen PK, Juhl E, Poulsen $\mathrm{H}$ and Tygstrup N: Prognostic value of Child-Turcotte criteria in medically treated cirrhosis. Hepatology 4: 430-435, 2010
27. Jia L, Binsheng F, Rongqin Z, et al: Value of ultrasonography in preoperative evaluation of vascular invasion and resectability of pancreatic cancer. Chin J Hepatic Surg, 410-413, 2018.

28. Kun Z, Dimitrov DD, Andreev TV, Liang W, Feradova H, Gorchev AG, Hui Z and Zhibiao W: One-year survival of inoperable pancreatic cancer treated by high intensity focused ultrasound: A retrospective multiple-center clinical trial in China and Bulgaria. J Chongqing Med Univ 40: 378-382, 2015.

29. Wu F, Wang ZB, Zhu H, Chen WZ, Zou JZ, Bai J, Li KQ, Jin CB, Xie FL and Su HB: Feasibility of US-guided high-intensity focused ultrasound treatment in patients with advanced pancreatic cancer: Initial experience. Radiology 236: 1034-1040, 2005.

30. Bin HU, Wei LV and Dan W: Effect of HIFU on relieving the pain of advanced pancreatic cancer. J Hepatopancreatobiliary Surg, 105-108, 2014.

31. Sung HY, Jung SE, Cho SH, Zhou K, Han JY, Han ST, Kim JI, Kim JK, Choi JY, Yoon SK, et al: Long-term outcome of high-intensity focused ultrasound in advanced pancreatic cancer. Pancreas 40: 1080-1086, 2011.

32. Vidal-Jove J, Perich E, Jaen A and Castillo Manuel AD: Ultrasound guided high intensity focused ultrasound (USgHIFU) for malignant tumors: Survival advantage in stage III and IV pancreatic cancer. J Ther Ultrasound 3 (Suppl 1): O79, 2015.

33. Zhao J, Zhao F, Shi Y, Deng Y, Hu X and Shen H: The efficacy of a new high intensity focused ultrasound therapy for locally advanced pancreatic cancer. J Cancer Res Clin Oncol 143: 2105-2111, 2017.

34. Ji Y, Zhang Y, Zhu J, Zhu L, Zhu Y, Hu K and Zhao H: Response of patients with locally advanced pancreatic adenocarcinoma to high-intensity focused ultrasound treatment: A single-center, prospective, case series in China. Cancer Manag Res 10: 4439-4446, 2018.

35. Zhang L, Zhu H, Jin C, Zhou K, Li K, Su H, Chen W, Bai J and Wang Z: High-intensity focused ultrasound (HIFU): Effective and safe therapy for hepatocellular carcinoma adjacent to major hepatic veins. Eur Radiol 19: 437-445, 2009.

36. Jiang F, He M, Liu Y, Huang X, Zhang L, Bai J and Wang Z: Pathological observation after MRI guided high intensity focused ultrasound therapy for ablating the liver tissues adjacent to goat portal vein. Sheng Wu Yi Xue Gong Cheng Xue Za Zhi 28: 666-669, 2011 (In Chinese).

37. Mingdong J, Jin B and Zhibiao W: Hemodynamic changes of the atheromatous abdominal aorta of rabbits treated with high intensity focused ultrasound. J Clin Ultrasound Med, 196-198, 2004.

38. Marik PE, Monnet X and Teboul JL: Hemodynamic parameters to guide fluid therapy. Ann Intensive Care 1: 1, 2011

39. Jung SE, Cho SH, Jang JH and Han JY: High-intensity focused ultrasound ablation in hepatic and pancreatic cancer: Complications. Abdom Imaging 36: 185-195, 2011.

40. Gao HF, Wang K, Meng ZQ, Chen Z, Lin JH, Zhou ZH, Wang P, Shi WD and Sheng YH: High intensity focused ultrasound treatment for patients with local advanced pancreatic cancer. Hepatogastroenterology 60: 1906-1910, 2013.

41. Wang G and Zhou D: Preoperative ultrasound ablation for borderline resectable pancreatic cancer: A report of 30 cases. Ultrason Sonochem 27: 694-702, 2015.

42. Girelli R, Frigerio I, Salvia R, Barbi E, Tinazzi Martini P and Bassi C: Feasibility and safety of radiofrequency ablation for locally advanced pancreatic cancer. Br J Surg 97: 220-225, 2010.

43. Girelli R, Frigerio I, Giardino A, Regi P, Gobbo S, Malleo G, Salvia R and Bassi C: Results of 100 pancreatic radiofrequency ablations in the context of a multimodal strategy for stage III ductal adenocarcinoma. Langenbecks Arch Surg 398: 63-69, 2013.

44. Mansson C, Brahmstaedt R, Nilsson A, Nygren P and Karlson BM: Percutaneous irreversible electroporation for treatment of locally advanced pancreatic cancer following chemotherapy or radiochemotherapy. Eur J Surg Oncol 42: 1401-1406, 2016.

45. Yan L, Chen YL, Su M, Liu T, Xu K, Liang F, Gu WQ and Lu SC: A single-institution experience with open irreversible electroporation for locally advanced pancreatic carcinoma. Chin Med J (Engl) 129: 2920-2925, 2016.

46. Scheffer HJ, Vroomen LG, de Jong MC, Melenhorst MC, Zonderhuis BM, Daams F, Vogel JA, Besselink MG, van Kuijk C, Witvliet $\mathrm{J}$, et al: Ablation of locally advanced pancreatic cancer with percutaneous irreversible electroporation: Results of the phase I/II PANFIRE study. Radiology 282: 585-597, 2017.

47. Kluger MD, Epelboym I, Schrope BA, Mahendraraj K, Hecht EM, Susman J, Weintraub JL and Chabot JA: Single-institution experience with irreversible electroporation for T4 pancreatic cancer: First 50 patients. Ann Surg Oncol 23: 1736-1743, 2016. 
48. Narayanan G, Hosein PJ, Beulaygue IC, Froud T, Scheffer HJ, Venkat SR, Echenique AM, Hevert EC, Livingstone AS, Rocha-Lima CM, et al: Percutaneous image-guided irreversible electroporation for the treatment of unresectable, locally advanced pancreatic adenocarcinoma. J Vasc Interv Radiol 28: 342-348, 2017.

49. Frigerio I, Girelli R, Giardino A, Regi P, Salvia R and Bassi C: Short term chemotherapy followed by radiofrequency ablation in stage III pancreatic cancer: Results from a single center. J Hepatobiliary Pancreat Sci 20: 574-577, 2013.

50. D'Onofrio M, Crosara S, De Robertis R, Butturini G, Salvia R, Paiella S, Bassi C and Mucelli RP: Percutaneous radiofrequency ablation of unresectable locally advanced pancreatic cancer: Preliminary results. Technol Cancer Res Treat 16: 285-294, 2017
51. Zhang Y, Shi J, Zeng J, Alnagger M, Zhou L, Fang G, Long X, Pan Z, Li Y, Chen J, et al: Percutaneous irreversible electroporation for ablation of locally advanced pancreatic cancer: Experience From a Chinese institution. Pancreas 46: e12-e14, 2017.

This work is licensed under a Creative Commons Attribution-NonCommercial-NoDerivatives 4.0 International (CC BY-NC-ND 4.0) License. 